\title{
The Knowledge or Random Guessing Model for Matching Tests
}

\author{
A. H. G. S. van der Ven and F. M. Gremmen \\ University of Nijmegen
}

The knowledge or random guessing (KRG) model was applied to matching tests. A matching test typically consists of two lists of alternatives. The response alternatives in the first list might consist of several terms to be defined, and the question alternatives in the second list would then consist of the definitions. Examinees are instructed to match the question alternatives to the response alternatives. According to the KRG model, if an examinee knows the correct answer, the correct answer will be chosen; however, if the examinee does not know the correct match, he/she will select the question alternative by guessing at random. Reliability formulas for the number of correct matchings based on the KRG model are given by Zimmerman and Williams (1982). Before applying these formulas, an appropriate statistical test

\begin{abstract}
should be used to test whether the model holds. A goodness-of-fit test is developed that is especially sensitive to the assumption of random guessing. Moreover, a simplified version of the model is presented in which the alternatives are ordered according to a Guttman scale. Three examples are given in which the model is applied to real data. It appears that in many cases examinees use coping strategies that violate the assumption of random guessing. A suggestion is made for the development of a somewhat more complex model that takes into account examinee coping strategies and that can be considered an extension of the KRG model. Index terms: achievement testing, guessing in matching tests, knowledge or random guessing model, matching tests.
\end{abstract}

Examinees taking multiple-choice tests can select correct answers to items that they could not have answered in an open-ended format. This fact is usually referred to as "the guessing problem" and has been the subject of a large body of theoretical and empirical research (for partial reviews of this work, see, e.g., Abu-Sayf, 1977; Diamond \& Evans, 1973; Hutchinson, 1982). This paper is related to the effects of chance success in matching tests. A matching test (see Wood, 1960, p. 27) typically consists of a list of response alternatives followed by a list of question alternatives. For example, the response alternatives may be the classes of vertebrates:

\section{Fish 2. Bird 3. Mammal 4. Reptile 5. Amphibian}

and question alternatives may consist of a list of the following animals:
a. Lion
b. Frog
c. Trout
d. Turtle
e. Eagle

This constitutes a five-alternative matching test. The examinee is instructed to match the animals to the vertebrate classes. If the number of question alternatives is larger than the number of response alternatives, it is called multiple matching. Recently, Budescu (1988) reported an empirical study on the feasibility of the multiple-matching test format. The present study, however, is primarily devoted to simple matching in which the number of question alternatives is equal to the number of response alternatives. However, the results can be generalized to multiple matching.

Reliability formulas for the number of correct matchings based on the well-known knowledge or

APPLIED PSYCHOLOGICAL MEASUREMENT

Vol. 16, No. 2, June 1992, pp. 177-194

(C) Copyright 1992 Applied Psychological Measurement Inc.

0146-6216/92/020177-18\$2.15 
random guessing (KRG) model (Lord \& Novick, 1968, p. 303, chap. 14.1-14.4) are given by Zimmerman and Williams (1982). They present $\rho_{X X^{\prime}}=1-[1 / \operatorname{Var}(X)]$, where $X$ is the number of correct matchings, as a lower-bound estimate for the reliability. This formula only applies when $k \leq n-2$ for all examinees, where $k$ is the number of correct matchings that were known and $n$ the number of matchings to be made. According to Zimmerman and Williams (p. 977), a more accurate formula is

$\rho_{x x^{\prime}}=1-1 / \operatorname{Var}(X)+N_{k} /[N \operatorname{Var}(X)]$,

where $N$ is the total number of examinees and $N_{k}$ is the number of examinees for which $k$ is equal to $n$ or $n-1$. Rewriting this formula leads to

$\rho_{x x^{\prime}}=\left[\operatorname{Var}(X)-\left(N-N_{k}\right) / N\right] / \operatorname{Var}(X)$.

It is evident that the formulas given by Zimmerman and Williams (1982) only apply if the KRG model holds. The KRG model is a very restrictive model, because the assumption of equal preferences for the unknown alternatives usually is not met. In most cases, examinees use coping strategies when they do not know the correct alternative. These strategies, however, are inconsistent with the assumption of random guessing. Therefore, it is necessary to test the model statistically before using these reliability coefficients. This article presents a statistical test of the KRG model, and a simplified version of the model is introduced in which it is assumed that the alternatives can be ordered according to a Guttman scale.

\section{Mathematical Derivations}

According to the KRG model, an examinee either knows or does not know the correct alternative. Let

$n$ be the total number of alternatives,

$k$ the number of known alternatives,

$X$ the number of correct alternatives, and

$G$ the number of altermatives that are correctly guessed;

then $X=k+G$, where $X$ and $G$ are stochastic variables and $k$ is a fixed constant. The probability distribution of $X$ and $G$ are related as follows:

$P(\mathbb{X}=x \mid n, k) \Leftrightarrow P(G=g \mid n-k) \quad$,

where $g=x-k$ and $x=0,1,2, \ldots, n$. When no alternative is known, $k=0$ and $X$ and $G$ coincide.

\section{The Probability Distribution of the Number of Correct Matchings}

The probability distribution of $X$ will be derived for $k=0$ (i.e., when the distributions of $X$ and $G$ coincide). Let $A$ be the number of sequences with $g$ correct matchings. For each of the $g$ correct matchings, the remaining $n-g$ are completely unmatched. There are $\left(\begin{array}{l}n \\ g\end{array}\right)$ possible ways to obtain a set of $g$ correct matchings in $n$ alternatives. Therefore,

$A(n, g)=\left(\begin{array}{l}n \\ g\end{array}\right) A(n-g, 0)$.

According to Kendall and Stuart (1963, pp. 184-185, example 3),

$A(n, g)=(n-g) ! \sum_{i=2}^{n-g}(-1)^{i} / i !$ for $\mathrm{n} \geq 2 ;$

therefore, 
$A(n, g)=\frac{n !}{g !} \sum_{i=2}^{n-g}(-1)^{i} / i ! \quad$ for $n \geq 2$.

The probability distribution of $G$ can now be described as

$P(G=g)=\left\{\begin{array}{lll}A(n, g) / n ! & \text { for } g=0, n-2 & A(n, n-1)=0 \\ 0 & \text { for } g=n-1 & A(n, n)=1 \\ 1 / n ! & \text { for } g=n . & \end{array}\right.$

In the limit for $\mathrm{n} \rightarrow \infty$, the distribution of $G$ approximates the Poisson distribution with expectation equal to 1. The approximation is satisfactory for $n=5$. Expectation and variance are both equal to 1 , regardless of the number of alternatives $(n)$.

Usually, the examinee does know some of the alternatives provided in the item. The probability distribution of the number of correct alternatives, $X$, given that $k$ alternatives are known, is given by:

$P(X=x \mid k)=\left\{\begin{array}{ll}A(n-k, x-k) /(n-k) ! & \text { for } k \leq x \leq n-2 \\ 0 & \text { for } x=n-1 \\ 1 /(n-k) ! & \text { for } x=n .\end{array}\right.$,

Because the number of correct alternatives is given by $X=k+G$, the expectation of $X$ can be described as

$\mathrm{E}(X \mid k)= \begin{cases}k+1 & \text { for } k \leq n-1 \\ k & \text { for } k=n\end{cases}$

and the variance as

$\operatorname{Var}(X \mid k)=\left\{\begin{array}{ll}1 & \text { for } k \leq n-2 \\ 0 & \text { for } k \geq n-1\end{array}\right.$.

These formulas are sufficient to derive a formula for the reliability of $X$, the number of correct alternatives (see Zimmerman \& Williams, 1982).

\section{A Statistical Test of the Model}

The statistical test described below is based on the matrix $O$ of observed frequencies. The element $o_{i j}$ denotes the observed number of examinees that have given question alternative $i$ to response alternative $j$. The matrix $\mathbb{O}$ is compared with the corresponding matrix of expected frequencies $\mathbb{E}$. For the derivation of the elements $e_{i j}$, the indices $i$ and $j$ are evaluated by setting $j$, which belongs to a certain question alternative, equal to $i$, which belongs to the corresponding correct question alternative. $K_{i}=1$ denotes that alternative $i$ is known; $K_{i}=0$ denotes that alternative $i$ is not known. An elementary event is a pattern of known and not-known alternatives. For example, for $n=5$, values of $K_{i}$ might be

$\left(K_{1}=0, K_{2}=1, K_{3}=0, K_{4}=0, K_{5}=1\right)$.

Therefore, an elementary event can be described as a series of $1 \mathrm{~s}$ and $0 \mathrm{~s}$. The number of possible elementary events is equal to $2^{\prime \prime}$. Let

$\pi\left(i_{1}, i_{2}, \ldots, i_{n}\right) \equiv \mathbb{P}\left(K_{1}=i_{1}, K_{2}=i_{2}, \ldots, K_{n}=i_{n}\right)$,

where $i_{k}=0$ or $i_{k}=1$. Let $M_{i j}$ denote that question alternative $i$ is matched to response alternative 
$j$. The probability $M_{i j}$ can now be expressed in terms of the probabilities $\pi$. Consider the probability that $M_{i j}$ will occur given a certain pattern of known and not-known alternatives. Two different situations are distinguished: $i=j$ and $i \neq j$. For $i=j$, the conditional probability is equal to 1 if $K_{i}=1$ :

$P\left(M_{i i} \mid K_{1}=i_{1}, K_{2}=i_{2}, \ldots, K_{i}=1, \ldots, K_{n}=i_{n}\right)=1$.

If $K_{i}=0$, then the examinee will select alternative $i$ by random guessing from the set remaining alternatives that are not known:

$P\left(M_{i i} \mid K_{1}=i_{1}, K_{2}=i_{2}, \ldots, K_{i}=0, \ldots, K_{n}=i_{n}\right)=\frac{1}{n-\sum_{k=1}^{n} i_{k}}$.

For $i \neq j$, the conditional probability is equal to 0 if $K_{i}=1$ :

$P\left(M_{i j} \mid K_{1}=i_{1}, K_{2}=i_{2}, \ldots, K_{i}=1, \ldots, K_{n}=i_{n}\right)=0$,

and also if $K_{j}=1$ :

$P\left(M_{i j} \mid K_{1}=i_{1}, K_{2}=i_{2}, \ldots, K_{j}=1, \ldots, K_{n}=i_{n}\right)=0$.

However, if $K_{i}=0$ and $K_{j}=0$, then the examinee will guess at random:

$P\left(M_{i j} \mid K_{1}=i_{1}, \ldots, K_{i}=0, \ldots, K_{j}=0, \ldots, K_{n}=i_{n}\right)=\frac{1}{n-\sum_{k=1}^{n} i_{k}}$.

The unconditional probability $P\left(M_{i j}\right)$ can now be written as a sum over the $2^{n}$ configurations $\left(i_{1}, i_{2}\right.$, $\ldots, i_{n}$ ) of products of the above conditional probabilities for $M_{i j}$ and the probabilities $\pi$ :

$P\left(M_{i j}\right)=\sum P\left(M_{i j} \mid K_{1}=i_{1}, K_{2}=i_{2}, \ldots, K_{n}=i_{n}\right) \pi\left(i_{1}, i_{2}, \ldots, i_{n}\right)$.

For $i \neq j$, at most $2(n-2)$ patterns are not equal to 0 due to Equations 15 and 16 . The computation of $P\left(M_{11}\right)$ and $P\left(M_{12}\right)$ for $n=3$ is illustrated in Table 1.

Because the probabilities $\pi$ sum to 1 , the number of unknown parameters is equal to $2^{n}-1$. The matrix $\mathbb{P}(M)$ also has the property of symmetry. This property could be used for a $\chi^{2}$ goodness-offit test of the model comparing the observed frequencies $o_{i j}$ with their corresponding expected frequencies $e_{i j}$. If expected frequencies are computed as follows:

$e_{i j}=\frac{o_{i j}+o_{j i}}{2}$

then

$\chi_{1}^{2}=\sum_{i=1}^{n-1} \sum_{j=i+1}^{n} \frac{\left(o_{i j}-e_{i j}\right)^{2}}{e_{i j}}$

would have a $\chi^{2}$ distribution with $1 / 2(n-1)(n-2)$ degrees of freedom $(d f)$.

The formula for $d f$ requires some clarification. The sum of the frequencies $o_{i j}$ in each column and in each row equals the number of examinees. The null hypothesis is that the frequencies in the offdiagonal elements of the $n \times n$ table are symmetric. Under the alternative hypothesis, there are 
Table 1

Computation of $P\left(M_{11}\right)$ and $P\left(M_{12}\right)$ for $n=3$

\begin{tabular}{rr}
\hline \hline $1 / 3 \pi(0,0,0)$ & $1 / 3 \pi(0,0,0)$ \\
$1 / 2 \pi(0,0,1)$ & $1 / 2 \pi(0,0,1)$ \\
$1 / 2 \pi(0,1,0)$ & $0 \pi(0,1,0)$ \\
$1 \pi(1,0,0)$ & $0 \pi(1,0,0)$ \\
$1 \pi(0,1,1)$ & $0 \pi(0,1,1)$ \\
$1 \pi(1,0,1)$ & $0 \pi(1,0,1)$ \\
$1 \pi(1,1,0)$ & $0 \pi(1,1,0)$ \\
$+1 \pi(1,1,1)$ & $+0 \pi(1,1,1)$ \\
\hline$P\left(M_{11}\right)=\ldots$ & $P\left(M_{12}\right)=\ldots$ \\
\hline
\end{tabular}

$(n-1)(n-2)$ varying off-diagonal elements, because the frequencies in the last row and last column can be determined in advance. Under the null hypothesis, there are $1 / 2(n-1)(n-2)$ varying frequencies. Therefore, the $d f$ equals $(n-1)(n-2)-1 / 2(n-1)(n-2)$.

The actual sampling distribution of $\chi_{1}^{2}$ may deviate considerably from a $\chi^{2}$ distribution with $1 / 2(n-1)(n-2) d f$ for two reasons. A $\chi^{2}$ test assumes independence of observations, which is not the case here. The total number of observations in the matrix $O$ is equal to $n N$, where $n$ is the number of alternatives and $N$ is the number of examinees in the sample. Although it can be assumed that $N$ people respond independently, it is unrealistic to assume that a given examinee responds independently to $n$ items. Secondly, some of the cells $o_{i j}$ in the matrix $O$ may be symmetric, not due to random sampling, but of necessity. For example, suppose that the population of examinees can be described by the following set of probabilities $\pi\left(i_{1}, i_{2}, \ldots, i_{n}\right)$ where $n=6$ :

$$
\begin{aligned}
& \pi(1,0,0,0,1,1)=.20 \\
& \pi(1,0,1,1,0,1)=.20 \\
& \pi(0,0,0,0,1,0)=.20 \\
& \pi(0,0,1,0,1,1)=.20 \\
& \pi(1,1,1,1,0,0)=.20 .
\end{aligned}
$$

Given this set of probabilities, the observed frequencies $o_{1,5}, o_{3,5}$, and $o_{4,5}$, as well as their symmetric counterparts, $o_{5,1}, o_{5,3}$, and $o_{5,4}$, must be equal to 0 , because it never happens that both of the items concerned are unknown. The observed frequencies $o_{2,5}$ and $o_{5,2}$ are symmetric, because when both items are unknown then all other items are known. This is also the case for the observed frequencies $o_{5,6}$ and $o_{6,5}$. Therefore, for the computation of $d f$, it is necessary to take into account the symmetric frequencies in the off-diagonal elements of the matrix $\mathbf{O}$ that occur by necessity. However, in the case of real data, symmetric frequencies may be a result of random sampling. For example, the matrix of observed frequencies $o_{i j}$ shown in Table 2 may be generated from the above set of probabilities, with $.5 \times(5-1)(5-2)=6-4=2 d f$.

The frequencies in parentheses in Table 2 are symmetric by necessity, but other frequencies occur that are also symmetric. Therefore, when using actual observations, it is impossible to know which symmetries occur by necessity and which occur by accident.

It was decided to take into account all symmetric frequencies for the computation of the $d f$. For the computation of $d f$, the number of symmetric rows in 0 can never be equal to $n-1$. If no symmetric frequencies occur, $d f$ is equal to $1 / 2(n-1)(n-2)$. If symmetric frequencies occur, then a complete row could be symmetric, and consequently a complete column also. In that case, $d f$ is equal 
Table 2

The Matrix of Observed Frequencies $o_{i j}$ Generated from the Set of Probabilities $\pi\left(i_{1}, i_{2}, \ldots, i_{n}\right)$

\begin{tabular}{cccccc}
\hline \hline 38 & 3 & 4 & 2 & $(0)$ & 3 \\
2 & 28 & 4 & $\{8\}$ & $(6)$ & $\{2\}$ \\
5 & 3 & 33 & $\{7\}$ & $(0)$ & $\{2\}$ \\
3 & $\{8\}$ & $\{7\}$ & 31 & $(0)$ & 1 \\
$(0)$ & $(6)$ & $(0)$ & $(0)$ & 35 & $(9)$ \\
2 & $\{2\}$ & $\{2\}$ & 2 & $(9)$ & 33 \\
\hline
\end{tabular}

to $1 / 2(r-1)(r-2)$, where $r$ is the number of rows (columns) in the matrix, in which the symmetric rows and columns are omitted. This is repeated until there are no more symmetric rows. If this leads to less than three rows (columns), then $O$ is symmetric, and $d f=0$. In the matrix that remains (in which all symmetric rows and columns have been removed), symmetric frequencies could still occur. For each symmetry, one $d f$ is subtracted from $1 / 2(r-1)(r-2)$. Because no distinction is made between necessary symmetries and accidental symmetries (i.e., symmetries that emerge as a result of random sampling), the actual distribution of $\chi_{1}^{2}$ could differ from the $\chi^{2}$ distribution. The distribution of $\chi_{1}^{2}$ could also depart from a $\chi^{2}$ distribution because of the lack of independence of observations mentioned above. A series of simulation studies was conducted in order to evaluate these deviations.

\section{Simulation of $\chi_{1}^{2}$}

Method. Three series of simulation studies were performed. Each series consisted of seven studies dependent on the number of alternatives, which varied from 3 to 9 . In each simulation study, the probabilities $\pi\left(i_{1}, i_{2}, \ldots, i_{n}\right)$ were determined as follows. First, 10 patterns $\left(i_{1}, i_{2}, \ldots, i_{n}\right)$ were selected at random with replacement from the set of all $2^{n}$ patterns, each with probability $1 /\left(2^{n}\right)$. Subsequently, depending on the series, the probabilities $\pi(\cdot)$ were assigned to the patterns $\left(i_{1}, i_{2}, \ldots, i_{n}\right)$ in the following way. In the first series, the probabilities $\pi(\cdot)$ were homogeneous with $\pi(\cdot)=.10$; in the second series, the probabilities were linear with

$\pi(\cdot)=\frac{k}{.5 \times 10 \times 11}$, where $k=1,2, \ldots, 10 ;$

and in the third series, exponential with

$\pi(\cdot)=\frac{100^{k / 10}}{\sum_{k=1}^{10} 100^{k / 10}}$, where $k=1,2, \ldots, 10$.

Each simulation study consisted of 400 samples of 1,000 simulated examinees (simulees). Each simulee possessed a knowledge pattern $\left(i_{1}, i_{2}, \ldots, i_{n}\right)$, which was randomly sampled from the 10 patterns discussed above, each with a probability $\pi(\cdot) .1,000$ simulees were selected in order to maintain expected frequencies larger than 5. For each simulee, a match was generated according to the model. On the basis of these 1,000 matches, the frequencies of simulees selecting response alternative $j$ to question alternative $i$ were computed. This resulted in a matrix of observed frequencies $O$. Next, the value of $\chi_{1}^{2}$ was computed. This was repeated 400 times. In other words, $400 \chi_{1}^{2} \mathrm{~s}$ were simulated, each based on 1,000 simulees. For each of the $400 \chi_{1}^{2} \mathrm{~s}$, the same 10 knowledge patterns were used with probabilities $\pi(\cdot)$. 
Table 3

Simulation Results For Knowledge Patterns With a Random Structure, Equal $\pi(\cdot)$, and 9 Alternatives: $d f$, Mean $\chi_{1}^{2}$, Number of Cases with $d f$ Degrees of Freedom $\left(f_{d f}\right), .05$ and .01 Critical $\chi^{2}$ Values, and Number of Cases in Which the .05 $\left(f_{05}\right)$ and .01 $\left(f_{\text {.01 }}\right)$ Critical $\chi^{2}$ Values Were Exceeded

\begin{tabular}{rrrrrrr}
\hline$d f$ & $f_{d f}$ & Mean $\chi_{1}^{2}$ & $\chi_{.05}^{2}$ & $f_{.05}$ & $\chi_{.01}^{2}$ & $f_{.01}$ \\
\hline 18 & 2 & 21.05 & 28.87 & 0 & 34.81 & 0 \\
19 & 4 & 17.53 & 30.14 & 0 & 36.19 & 0 \\
20 & 19 & 21.20 & 31.41 & 2 & 37.57 & 0 \\
21 & 57 & 21.83 & 32.67 & 5 & 38.93 & 2 \\
22 & 93 & 23.06 & 33.92 & 6 & 40.29 & 3 \\
23 & 132 & 23.55 & 35.17 & 8 & 41.64 & 2 \\
24 & 93 & 25.80 & 36.42 & 12 & 42.98 & 1 \\
\hline
\end{tabular}

It was argued above that in the case of actual observations it is impossible to know which symmetries occur by necessity and which occur by accident; therefore, $d f$ were subtracted for all symmetric frequencies. Consequently, within a simulation study, the $d f$ varied. In each simulation study, the proportion of cases was computed in which the simulated $\chi_{1}^{2}$ exceeded both the $5 \%$ and $1 \%$ critical $\chi^{2}$ values. The way in which these proportions were computed in the case of varying $d f$ is exemplified using the simulation study based on knowledge patterns with a random structure with equal $\pi(\cdot)$ and 9 alternatives (see $n=9$ in Table 4). The various $d f$ that occurred in that particular simulation study are given in the first column of Table 3.

Results. The critical value of $\chi^{2}$ at the $5 \%$ level for $21 d f$ is equal to 32.67 , and in 5 out of 57 cases the value of the simulated $\chi_{1}^{2}$ exceeded that value. The computed $\chi_{1}^{2}$ exceeded the critical $\chi^{2}$ at the .05 level in 33 instances (out of 400), a proportion of .0825 (rounded to .08 in Table 4). Similar$1 y$, the computed $\chi_{1}^{2}$ exceeded the critical $\chi^{2}$ at the .01 level in 8 instances, resulting in a proportion of .02 .

The results of the 21 simulation studies are given in Table 4, based on the product of seven levels of alternatives (varied from 3 to 9) and three models (homogeneous, linear, and exponential). Because the $d f$ varied within each simulation study, the mean and variance of the number of $d f$ are given in Table 4 . The ratio between the mean $\chi_{1}^{2}$ and the mean $d f$ usually is somewhat above 1.0 , indicating that the mean $\chi_{1}^{2}$ is slightly larger than its corresponding $d f$. The same holds for the ratio between the variance of $\chi_{1}^{2}$ divided by 2 and the mean $d f$. The mean $\chi_{1}^{2}$ should be equal to the mean $d f$, and the variance of $\chi_{1}^{2}$ to two times the mean $d f$. The results indicate that the actual distribution of $\chi_{1}^{2}$ deviates somewhat from the expected $\chi^{2}$ distribution. This conclusion also holds with respect to $p_{.05}$ and $p_{.01}$, the proportions of cases in which $\chi_{1}^{2}$ exceeded the $5 \%$ and $1 \%$ critical $\chi^{2}$. The ratio of the variance of $\chi_{1}^{2} / 2$ and the mean of $\chi_{1}^{2}$ was also somewhat larger than 1.0 .

The results of the simulation studies with equal $\pi(\cdot)$ and 3 alternatives (Table 4) require some explanation. In that study, the number of $d f$ was equal to 0 for all simulations that resulted in a mean $d f$ of 0 and a variance of 0 . All simulated data matrices were completely symmetric. This occurred because the knowledge pattern $(0,0,0)$ did not occur in the set of 10 knowledge patterns. In this situation, all the cells were symmetric by necessity for reasons discussed above.

The 10 patterns $\left(i_{1}, i_{2}, \ldots, i_{n}\right)$ in these simulation studies were selected at random with replacement from the set of all $2^{n}$ patterns. No structure can be expected in the set of 10 patterns. It could be argued that if any structure was present in the 10 patterns, this could have some effect on the distribution of $\chi_{1}^{2}$. The number of structured sets of patterns might be very large. Some selection 


\section{Volume 16 Number 2 June 1992
184 APPLIED PSYCHOLOGICAL MEASUREMENT}

Table 4

Simulation Results for Knowledge Patterns With Random Structures: Number of Alternatives $(n)$, Mean and Variance of $d f$ and $\chi_{1}^{2}$ and Related Ratios, and Proportion of Cases in Which $\chi_{1}^{2}$ Exceeded the $.05\left(p_{.05}\right)$ and $.01\left(p_{.01}\right)$ Critical Values

\begin{tabular}{|c|c|c|c|c|c|c|c|c|c|}
\hline \multirow[b]{2}{*}{$n$} & \multicolumn{2}{|c|}{$d f$} & \multicolumn{2}{|c|}{$\chi_{1}^{2}$} & \multirow{2}{*}{$\frac{\text { Mean } \chi_{1}^{2}}{\text { Mean } d f}$} & \multirow{2}{*}{$\frac{\operatorname{Var} \chi_{1}^{2} / 2}{\text { Mean } d f}$} & \multirow{2}{*}{$\frac{\operatorname{Var} \chi_{i}^{2} / 2}{\text { Mean } \chi_{1}^{2}}$} & \multirow[b]{2}{*}{$p_{.05}$} & \multirow[b]{2}{*}{$p_{.01}$} \\
\hline & Mean & Var & Mean & Var & & & & & \\
\hline \multicolumn{10}{|c|}{ Equal $\pi(\cdot)$} \\
\hline 3 & 1.00 & 0.00 & 0.00 & 0.00 & - & - & - & 0.00 & 0.00 \\
\hline 4 & 2.69 & .25 & 2.51 & 5.03 & .93 & .93 & 1.00 & .04 & .01 \\
\hline 5 & 5.55 & .40 & 5.24 & 9.56 & .94 & .86 & .91 & .03 & 0.00 \\
\hline 6 & 7.28 & .68 & 7.43 & 17.51 & 1.02 & 1.20 & 1.18 & .05 & .01 \\
\hline 7 & 13.07 & .78 & 13.58 & 26.49 & 1.04 & 1.01 & .98 & .06 & .01 \\
\hline 8 & 15.88 & 1.05 & 16.62 & 35.65 & 1.05 & 1.12 & 1.07 & .07 & .02 \\
\hline 9 & 22.51 & 1.51 & 23.53 & 53.35 & 1.05 & 1.19 & 1.13 & .08 & .02 \\
\hline \multicolumn{10}{|c|}{ Linear $\pi(\cdot)$} \\
\hline 3 & 1.00 & 0.00 & .85 & 1.42 & .85 & .71 & .84 & .03 & .01 \\
\hline 4 & 1.00 & 0.00 & 1.43 & 3.61 & 1.43 & 1.80 & 1.26 & .10 & .03 \\
\hline 5 & 3.70 & .30 & 3.87 & 8.89 & 1.05 & 1.20 & 1.15 & .06 & .01 \\
\hline 6 & 9.38 & .60 & 9.55 & 20.45 & 1.02 & 1.09 & 1.07 & .06 & .02 \\
\hline 7 & 14.08 & .83 & 14.20 & 30.89 & 1.01 & 1.10 & 1.09 & .07 & .02 \\
\hline 8 & 18.43 & 1.30 & 17.93 & 32.28 & .97 & .88 & .90 & .05 & 0.00 \\
\hline 9 & 20.17 & 1.77 & 21.13 & 40.96 & 1.05 & 1.02 & .97 & .07 & .02 \\
\hline \multicolumn{10}{|c|}{ Exponential $\pi(\cdot)$} \\
\hline 3 & 1.00 & 0.00 & 1.09 & 2.46 & 1.09 & 1.23 & 1.13 & .06 & .01 \\
\hline 4 & 2.64 & .25 & 2.73 & 5.27 & 1.03 & 1.00 & .96 & .06 & .01 \\
\hline 5 & 4.70 & 1.09 & 5.04 & 9.84 & 1.07 & 1.05 & .98 & .05 & .01 \\
\hline 6 & 9.18 & .78 & 9.96 & 22.53 & 1.08 & 1.23 & 1.13 & .09 & .02 \\
\hline 7 & 12.98 & 1.09 & 13.78 & 30.50 & 1.06 & 1.17 & 1.11 & .08 & .02 \\
\hline 8 & 18.99 & 1.86 & 20.06 & 39.29 & 1.06 & 1.03 & .98 & .06 & .01 \\
\hline 9 & 20.14 & 2.77 & 21.54 & 39.71 & 1.07 & .99 & .92 & .05 & .02 \\
\hline
\end{tabular}

is, therefore, needed in order to end up with a considerable reduction. According to Coombs' theory of data (Coombs, 1964; Van der Ven, 1980, chap. 8), the state of knowledge of an examinee about an alternative can be regarded as a relation between objects from two different sets (the set of examinees and the set of alternatives). The objects are represented by points on a line (the scale). Two different possible relations defined on the points are of importance. In some instances, the only thing that counts is whether a point is situated to the right of another point, which is called an order or dominance relation on points. The proximity of two points may also be relevant, which is called a proximity relation. If the objects are represented by points according to a dominance relation and can be ordered on a single dimension, then a permutation of examinees (rows) and alternatives (columns) exists such that the 0 s and $1 \mathrm{~s}$ in the matrix of knowledge patterns result in a triangular structure. For example, for six alternatives, the matrix given in the left portion of Table 5 may be obtained, in which the rows correspond to the simulees and the columns to the alternatives.

If the objects are represented by points according to a proximity relation and can be ordered on a single dimension, then a permutation of examinees (rows) and alternatives (columns) exists such that the $0 \mathrm{~s}$ and $1 \mathrm{~s}$ in the matrix of knowledge patterns result in a parallelogram structure. For example, for six alternatives the matrix given in the right portion of Table 5 may be obtained, in which the rows correspond to the simulees and the columns to the alternatives.

Two new series of simulation studies were performed-one with a triangular structure in the 
Table 5

Triangular and Parallelogram Knowledge Structures for 6 Alternatives

\begin{tabular}{ccc}
\hline Examinee & Triangular & Parallelogram \\
\hline A & $0,0,0,0,0,0$ & $1,0,0,0,0,0$ \\
B & $1,0,0,0,0,0$ & $1,1,0,0,0,0$ \\
C & $1,1,0,0,0,0$ & $1,1,1,0,0,0$ \\
D & $1,1,1,0,0,0$ & $0,1,1,1,0,0$ \\
E & $1,1,1,1,0,0$ & $0,0,1,1,1,0$ \\
F & $1,1,1,1,1,0$ & $0,0,0,1,1,1$ \\
G & $1,1,1,1,1,1$ & $0,0,0,0,1,1$ \\
H & & $0,0,0,0,0,1$ \\
\hline
\end{tabular}

knowledge patterns and one with a parallelogram structure. In order to preserve the structure in the knowledge patterns as much as possible, random selection of patterns is not used to establish the set of patterns to which the probabilities $\pi\left(i_{1}, i_{2}, \ldots, i_{n}\right)$ were assigned. For the simulation of the matches of a single examinee, each pattern was selected with a probability $\pi\left(i_{1}, i_{2}, \ldots, i_{n}\right)=1 / k$. In the triangular case $k=n+1$, and in the parallelogram case $k=n+2$. The overall results of the $21(7 \times 3)$ simulation studies are given in Table 6 .

The ratio of the mean $\chi_{1}^{2}$ to the mean $d f$ is systematically above 1.0 , indicating that the mean $\chi_{1}^{2}$ was larger than its corresponding $d f$. The same holds for the ratio between the variance of $\chi_{1}^{2} / 2$ and the mean $d f$. The actual distribution of $\chi_{1}^{2}$ deviates systematically from the expected $\chi^{2}$ distribution. The respective values of $p_{.05}$ and $p_{.01}$ result in the same conclusion as in the previous studies. The table shows that $p_{.05}$ is approximately .05 larger than it should be and that $p_{.01}$ is approximately .01 larger than it should be.

These simulations demonstrate that $\chi_{1}^{2}$ does not have a $\chi^{2}$ distribution with $d f$ as described above. This is mainly the result of the violation of independence of observations, because $\chi_{1}^{2}$ deviated more

Table 6

Simulation Results for Knowledge Patterns With Triangular and Parallelogram Structures: Number of Alternatives $(n)$, Mean and Variance of $d f$ and $\chi_{1}^{2}$ and Related Ratios, and Proportion of Cases in Which $\chi_{1}^{2}$ Exceeded the $.05\left(p_{05}\right)$ and $.01\left(p_{01}\right)$ Critical Values

\begin{tabular}{|c|c|c|c|c|c|c|c|c|c|}
\hline \multirow[b]{2}{*}{$n$} & \multicolumn{2}{|c|}{$d f$} & \multicolumn{2}{|c|}{$\chi_{1}^{2}$} & \multirow{2}{*}{$\frac{\text { Mean } \chi_{t}^{2}}{\text { Mean } d f}$} & \multirow{2}{*}{$\frac{\operatorname{Var} \chi_{1}^{2} / 2}{\text { Mean } d f}$} & \multirow{2}{*}{$\frac{\operatorname{Var} \chi_{1}^{2} / 2}{\text { Mean } \chi_{1}^{2}}$} & \multirow[b]{2}{*}{$p_{.05}$} & \multirow[b]{2}{*}{$p_{.01}$} \\
\hline & Mean & Var & Mean & Var & & & & & \\
\hline \multicolumn{10}{|c|}{ Triangular Structure } \\
\hline 3 & 1.00 & 0.00 & 1.12 & 2.27 & 1.12 & 1.14 & 1.02 & .07 & .01 \\
\hline 4 & 2.72 & .23 & 3.42 & 8.29 & 1.26 & 1.52 & 1.21 & .09 & .02 \\
\hline 5 & 5.50 & .54 & 6.75 & 14.31 & 1.23 & 1.30 & 1.06 & .09 & .02 \\
\hline 6 & 9.25 & .76 & 10.77 & 23.60 & 1.16 & 1.28 & 1.10 & .09 & .02 \\
\hline 7 & 13.98 & .97 & 16.88 & 34.08 & 1.21 & 1.22 & 1.01 & .12 & .03 \\
\hline 8 & 19.55 & 1.21 & 22.49 & 48.88 & 1.15 & 1.25 & 1.09 & .12 & .02 \\
\hline 9 & 26.06 & 1.85 & 30.37 & 60.93 & 1.17 & 1.17 & 1.00 & .14 & .02 \\
\hline \multicolumn{10}{|c|}{ Parallelogram Structure } \\
\hline 3 & 1.00 & 0.00 & 0.00 & 0.00 & - & - & - & 0.00 & 0.00 \\
\hline 4 & 1.87 & .12 & 1.84 & 3.68 & .98 & .98 & 1.00 & .04 & 0.00 \\
\hline 5 & 4.59 & .40 & 4.86 & 10.64 & 1.06 & 1.16 & 1.09 & .06 & .01 \\
\hline 6 & 9.40 & .53 & 9.47 & 20.49 & 1.01 & 1.09 & 1.08 & .05 & .01 \\
\hline 7 & 14.22 & .75 & 15.03 & 36.85 & 1.06 & 1.30 & 1.23 & .08 & .03 \\
\hline 8 & 19.96 & 1.00 & 21.88 & 41.70 & 1.10 & 1.04 & .95 & .08 & .01 \\
\hline 9 & 26.78 & 1.20 & 29.89 & 65.52 & 1.12 & 1.22 & 1.10 & .11 & .03 \\
\hline
\end{tabular}


from its corresponding $d f$ in the case of the simulations with structured knowledge patterns than it did in the case of simulations with random patterns. At the same time, however, it may be concluded that the statistic can still be used as a conservative test of the model. If the model is rejected using the true sampling distribution of $\chi_{1}^{2}$, then it certainly would be rejected using the $\chi^{2}$ distribution.

\section{A Guttman Scale Version of the Model}

The matrix $\mathbf{P}(M)$ can be derived from the probabilities $\pi$, but the reverse is not true. In order to derive the probabilities $\pi$ from $\mathbf{P}(M)$, the model must be more restrictive. This can be achieved by assuming that some structure is present in the knowledge patterns. Two possible structures have been discussed above: one based on a dominance relation and one based on a proximity relation. Frequently, in practical applications, the alternatives are ordered (dominance relation) with respect to their knowledge structure. Therefore, the case in which a dominance relation holds will be elaborated.

If a dominance relation holds, the psychological objects (in this case the alternatives) form a Guttman scale. A typical example of an ordering in the knowledge elements is the computational operations in arithmetic: For example, multiplication implies addition; raising to a higher power implies multiplication; extraction of a root implies raising to a higher power, and so forth. Examinees who know the correct answer to multiplication problems also should know the correct answer to addition problems. Therefore, exercises taken from these domains might be ordered according to a Guttman scale. If the alternatives form a Guttman scale, then only $n+1$ knowledge patterns are possible, and the number of unknown probabilities $\pi$ is also equal to $n+1$. As a result, $\mathbb{P}(M)$ has some additional properties.

Let $\alpha_{j}$ be defined as follows:

$\alpha_{j} \equiv \pi\left(i_{1}, i_{2}, \ldots, i_{j}, i_{j+1}, \ldots, i_{n}\right)$,

where $i_{1}=1, i_{2}=1, \ldots, i_{j}=\mathbb{1}, i_{j}+\mathbb{1}=0, \ldots, i_{n}=0$, and

$\alpha_{0} \equiv \pi\left(i_{1}, i_{2}, \ldots, i_{j}, \ldots, i_{n}\right)$,

where $i_{j}=0$ for all $j$. For example, for $n=5$ :

$\alpha_{0}=\pi(0,0,0,0,0)$,

$\alpha_{1}=\pi(1,0,0,0,0)$,

$\alpha_{2}=\pi(1,1,0,0,0)$,

$\alpha_{3}=\pi(1,1,1,0,0)$,

$\alpha_{4}=\pi(1,1,1,1,0)$,

$\alpha_{s}=\pi(1,1,1,1,1)$.

Note that $\alpha_{4}$ and $\alpha_{5}$ should be thought of as amalgamated, because only the sum of $\alpha_{4}$ and $\alpha_{5}$ can be identified. If the elements of $\mathbb{P}(M)$ are rearranged so that the alternatives correspond to their order in the Guttman scale, then the probabilities $P\left(M_{i j}\right)$ can be written as follows ( $n=5$ is used as an example): 


$$
\begin{aligned}
& P\left(M_{11}\right)=1 / 5 \alpha_{0}+\alpha_{1}+\alpha_{2}+\alpha_{3}+\alpha_{4}+\alpha_{5} \\
& P\left(M_{22}\right)=1 / 5 \alpha_{0}+1 / 4 \alpha_{1}+\alpha_{2}+\alpha_{3}+\alpha_{4}+\alpha_{5} \\
& P\left(M_{33}\right)=1 / 5 \alpha_{0}+1 / 4 \alpha_{1}+1 / 3 \alpha_{2}+\alpha_{3}+\alpha_{4}+\alpha_{5} \\
& P\left(M_{44}\right)=1 / 5 \alpha_{0}+1 / 4 \alpha_{1}+1 / 3 \alpha_{2}+1 / 2 \alpha_{3}+\alpha_{4}+\alpha_{5} \\
& P\left(M_{55}\right)=1 / 5 \alpha_{0}+1 / 4 \alpha_{1}+1 / 3 \alpha_{2}+1 / 2 \alpha_{3}+\alpha_{4}+\alpha_{5} \\
& P\left(M_{45}\right)=1 / 5 \alpha_{0}+1 / 4 \alpha_{1}+1 / 3 \alpha_{2}+1 / 2 \alpha_{3} \\
& P\left(M_{35}\right)=1 / 5 \alpha_{0}+1 / 4 \alpha_{1}+1 / 3 \alpha_{2} \\
& P\left(M_{25}\right)=1 / 5 \alpha_{0}+1 / 4 \alpha_{1} \\
& P\left(M_{15}\right)=1 / 5 \alpha_{0},
\end{aligned}
$$

and $P\left(M_{34}\right)=P\left(M_{35}\right), P\left(M_{23}\right)=P\left(M_{24}\right)=P\left(M_{25}\right), P\left(M_{12}\right)=P\left(M_{13}\right)$, and so forth. This regularity applies to the general case with $n$ alternatives. Therefore, in each row of $\mathbf{P}(M)$ the off-diagonal elements to the right of the diagonal element are equal. Also, in the columns, the off-diagonal elements below the diagonal elements are equal. Therefore, a goodness-of-fit test of the Guttman scale version of the model can be conducted based on a comparison of observed and expected frequencies. The expected frequencies are computed as follows. Let $e_{i}$ equal the expected frequency for each offdiagonal cell in the row to the right of cell $(i, i)$ and in the column below cell $(i, i)$. Then

$e_{i}=\frac{\sum_{j=i+1}^{n} \frac{o_{i j}+o_{j i}}{2}}{n-i}$, for $i=1, \ldots, n-1$.

The sum of the expected frequencies in a row is equal to $N$, where $N$ is the number of examinees. Let $e_{i}^{\prime}$ be the expected frequency of the diagonal element in row $i$. Then

$$
\begin{aligned}
& e_{1}^{\prime}+(n-1) e_{1}=N \\
& e_{2}^{\prime}+(n-2) e_{2}+e_{1}=N \\
& e_{3}^{\prime}+(n-3) e_{3}+e_{2}+e_{1}=N \\
& \vdots \\
& e_{n}^{\prime}+(n-n) e_{n}+e_{1}+e_{2}+, \ldots,+e_{n-1}=N,
\end{aligned}
$$

or, more generally,

$e_{i}^{\prime}=N-(n-i) e_{i}-\sum_{j=1}^{i-1} e_{j}$.

The statistic $\chi_{2}^{2}$ is computed as follows:

$\chi_{2}^{2}=\sum_{i=1}^{n-1} \sum_{j=i+1}^{n}\left[\frac{\left(o_{i j}-e_{i}\right)^{2}}{e_{i}}+\frac{\left(o_{i j}-e_{i}\right)^{2}}{e_{i}}\right]+\sum_{i=1}^{n} \frac{\left(o_{i i}-e_{i}^{\prime}\right)^{2}}{e_{i}^{\prime}}$.

This statistic would have a $\chi^{2}$ distribution with $(n-1)(n-2) d f$. The alternative hypothesis implies $(n-1)(n-1)$ free frequencies, because the sum of the frequencies $o_{i j}$ in each column and in each row equals the number of examinees. The null hypothesis implies $(n-1)$ free frequencies, because for each row (or column), except the last row (or column), a frequency is assigned. Therefore, 
$d f=(n-1)(n-1)-(n-1)=(n-1)(n-2)$.

Similar to the general model, the actual sampling distribution of $\chi_{2}^{2}$ again may deviate considerably from a $\chi^{2}$ distribution with $(n-1)(n-2) d f$, for the same reasons. For example, suppose that the population of examinees can be described by the following set of probabilities $\pi\left(i_{1}, i_{2}, \ldots, i_{n}\right)$ where $n=6:$

$$
\begin{aligned}
& \pi(1,0,0,0,0,0)=.20 \\
& \pi(1,1,0,0,0,0)=.20 \\
& \pi(1,1,1,0,0,0)=.20 \\
& \pi(1,1,1,1,1,0)=.20 \\
& \pi(1,1,1,1,1,1)=.20
\end{aligned}
$$

Given this set of probabilities, the observed frequencies, $o_{1,2}, o_{1,3}, o_{1,4}, o_{1,5}$, and $o_{1,6}$, must be equal to 0 as well as their symmetric counterparts, $o_{2,1}, o_{3,1}, o_{4,1}, o_{5,1}$, and $o_{6,1}$, because it never happens that both of the items concerned are unknown. The frequencies are 0 by necessity. Because item 1 is known by all examinees, it is impossible that this item is matched to any of the other items. For the computation of $d f$, it is therefore necessary to take into account the 0 row and column frequencies of $O$, which occur by necessity, as for the previous model. Similarly, all 0 rows and columns were taken into account in the computation of $d f$. The final number of $d f$ was $(k-1)(k-2)$, where $k$ is the number of rows (columns) in the matrix in which the 0 rows and columns were omitted. In the computation of $d f$, no distinction was made between necessary and accidental 0 rows and columns. Therefore, the actual distribution of $\chi_{2}^{2}$ could be different from the $\chi^{2}$ distribution. The distribution of $\chi_{2}^{2}$ could also differ from the $\chi^{2}$ distribution with $d f=(k-1)(k-2)$ due to the lack of independence of observations mentioned above. A series of simulation studies was done in order to determine the extent of these deviations.

\section{Simulation of $\chi_{2}^{2}$}

Method. Three series of simulation studies were performed, using the same design as for the previous simulations. In each simulation study, the probabilities $\pi\left(i_{1}, i_{2}, \ldots, i_{n}\right)$ were determined as follows. First $r$ patterns $\left(i_{1}, i_{2}, \ldots, i_{n}\right)$ were selected at random without replacement from the set of all $n+1$ patterns, where $r=n$ for $n=3,4,5,6$ and $n-1$ for $n=7,8$, 9. Subsequently, depending on the series, the probabilities $\pi(\cdot)$ were assigned to the patterns $\left(i_{1}, i_{2}, \ldots, i_{n}\right)$ in the following way. In the first series, the probabilities $\pi(\cdot)$ were homogeneous with $\pi(\cdot)=1 / r$; in the second series, the probabilities $\pi(\cdot)$ were linear with

$\pi(\cdot)=\frac{k}{.5 r(r+1)}$, where $k=1,2, \ldots, r ;$

and in the third series, exponential with

$$
\pi(\cdot)=\frac{100^{k / r}}{\sum_{k=1}^{r} 100^{k / r}} \text {, where } k=1,2, \ldots, r
$$

Each examinee possessed a knowledge pattern $\left(i_{1}, i_{2}, \ldots, i_{n}\right)$, which was sampled randomly from the $r$ patterns discussed above, each with a probability $\pi(\cdot)$.

Results. The results of the 21 simulation studies are given in Table 7. As in the simulations with $\chi_{1}^{2}$, the ratio of the mean $\chi_{2}^{2}$ to the mean $d f$ was systematically above 1.0 - probably due to the above- 
Table 7

Simulation Results for Knowledge Patterns With Guttman Structures: Number of Alternatives $(n)$, Mean and Variance of $d f$ and $\chi_{2}^{2}$ and Related Ratios, and Proportion of Cases in Which $\chi_{2}^{2}$ Exceeded the $.05\left(p_{.05}\right)$ and $.01\left(p_{.01}\right)$ Critical Values

\begin{tabular}{|c|c|c|c|c|c|c|c|c|c|}
\hline \multirow[b]{2}{*}{$n$} & \multicolumn{2}{|c|}{$d f$} & \multicolumn{2}{|c|}{$\chi_{2}^{2}$} & \multirow{2}{*}{$\frac{\text { Mean } \chi_{2}^{2}}{\text { Mean } d f}$} & \multirow{2}{*}{$\frac{\operatorname{Var} \chi_{2}^{2} / 2}{\text { Mean } d f}$} & \multicolumn{3}{|l|}{$\operatorname{Var} \chi_{2}^{2} / 2$} \\
\hline & Mean & Var & Mean & Var & & & Mean $\chi_{2}^{2}$ & $p_{.05}$ & $p_{.0 \mathrm{i}}$ \\
\hline \multicolumn{10}{|c|}{ Equal $\pi(\cdot)$} \\
\hline 3 & 2.00 & 0.00 & 2.47 & 6.93 & 1.24 & 1.73 & 1.40 & .07 & .02 \\
\hline 4 & 6.00 & 0.00 & 6.75 & 18.04 & 1.13 & 1.50 & 1.34 & .09 & .03 \\
\hline 5 & 12.00 & 0.00 & 13.46 & 32.95 & 1.12 & 1.37 & 1.22 & .11 & .04 \\
\hline 6 & 20.00 & 0.00 & 21.22 & 44.60 & 1.06 & 1.11 & 1.05 & .07 & .02 \\
\hline 7 & 30.00 & 0.00 & 30.85 & 69.28 & 1.03 & 1.15 & 1.12 & .06 & .02 \\
\hline 8 & 42.00 & 0.00 & 44.77 & 94.07 & 1.07 & 1.12 & 1.05 & .08 & .03 \\
\hline 9 & 56.00 & 0.00 & 59.77 & 127.16 & 1.07 & 1.14 & 1.06 & .09 & .03 \\
\hline \multicolumn{10}{|c|}{ Linear $\pi(\cdot)$} \\
\hline 3 & 2.00 & 0.00 & 2.18 & 4.66 & 1.09 & 1.16 & 1.07 & .07 & .01 \\
\hline 4 & 6.00 & 0.00 & 6.77 & 16.20 & 1.13 & 1.35 & 1.20 & .08 & .03 \\
\hline 5 & 12.00 & 0.00 & 12.65 & 24.36 & 1.05 & 1.01 & .96 & .06 & .02 \\
\hline 6 & 20.00 & 0.00 & 21.27 & 46.94 & 1.06 & 1.17 & 1.10 & .09 & .02 \\
\hline 7 & 30.00 & 0.00 & 32.62 & 72.44 & 1.09 & 1.21 & 1.11 & .11 & .04 \\
\hline 8 & 30.00 & 0.00 & 32.05 & 69.94 & 1.07 & 1.17 & 1.09 & .07 & .02 \\
\hline 9 & 56.00 & 0.00 & 60.97 & 140.84 & 1.09 & 1.26 & 1.16 & .13 & .05 \\
\hline \multicolumn{10}{|c|}{ Exponential $\pi(\cdot)$} \\
\hline 3 & 0.00 & 0.00 & 0.00 & 0.00 & - & - & - & 0.00 & 0.00 \\
\hline 4 & 6.00 & 0.00 & 6.38 & 14.07 & 1.06 & 1.17 & 1.10 & .06 & .01 \\
\hline 5 & 6.00 & 0.00 & 6.11 & 13.85 & 1.02 & 1.15 & 1.13 & .07 & .01 \\
\hline 6 & 19.98 & .16 & 20.83 & 35.74 & 1.04 & .89 & .86 & .05 & .01 \\
\hline 7 & 30.00 & 0.00 & 31.91 & 67.68 & 1.06 & 1.13 & 1.06 & .08 & .02 \\
\hline 8 & 42.00 & 0.00 & 45.43 & 92.65 & 1.08 & 1.10 & 1.02 & .08 & .02 \\
\hline 9 & 55.96 & .49 & 57.51 & 124.02 & 1.03 & 1.11 & 1.08 & .08 & .02 \\
\hline
\end{tabular}

mentioned violation of independence of observations. The same holds for the ratio between the variance of $\chi_{2}^{2} / 2$ and the mean $d f$. Also, in the case of $\chi_{2}^{2}$, the actual sampling distribution deviated systematically from the expected $\chi^{2}$ distribution. However, because the actual sampling distribution of $\chi_{2}^{2}$ is somewhat shifted to the right compared to the expected $\chi^{2}$ distribution, the statistic $\chi_{2}^{2}$ still can be used as a conservative test of the model. If the model is rejected using the true sampling distribution of $\chi_{2}^{2}$, then it would be rejected when the $\chi^{2}$ distribution was used. Values of $p_{.05}$ and $p_{.01}$ were similar to those of the previous simulations. The $\chi_{1}^{2}$ statistic was also computed for these data. These results were very similar to those obtained with simulations based on knowledge patterns with a triangular structure.

\section{Example Applications}

Three different examples are illustrated. Examples 1 and 3 show the confounding effect of apparent coping strategies. In Example 1, examinees appeared to resort to different domains of knowledge to answer some questions, and in Example 3, the apparent similarity of the alternatives was used to select an answer. Example 2 is an application of the Guttman scale version of the model.

\section{Example 1: Schools in Psychology}

This example used a matching test that was administered to students $(N=270)$ as part of an examination in an introductory course on the history of psychology. In the textbook (Leahey, 1980), 
Table 8

Response Alternatives and Question Alternatives for Three Matching Tests

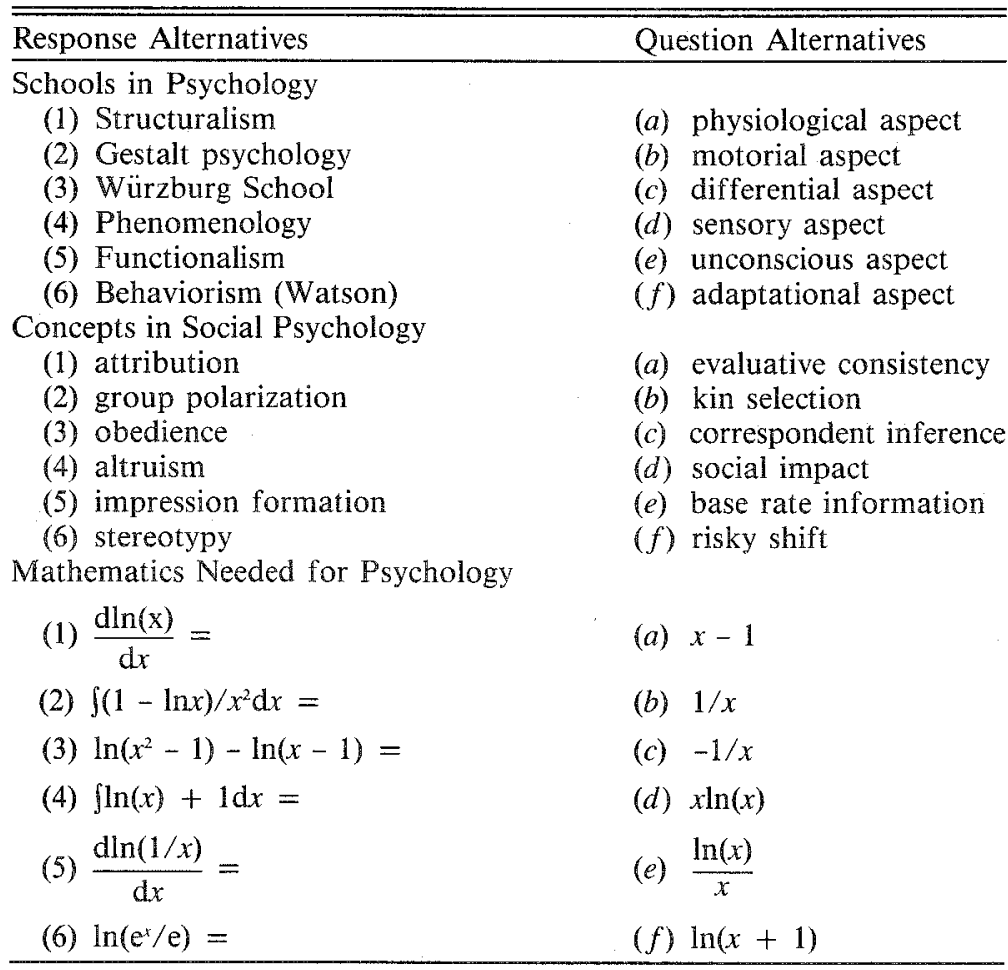

different schools of psychology are discussed. The response alternatives consisted of different schools of psychology, and the question alternatives contained the dominant aspects prevailing in these schools (see Table 8). Table 9 shows the frequencies of examinees who selected response alternative $j(j=$ $1,2, \ldots, 6)$ to question alternative $i(i=a, b, \ldots, f)$. Application of the symmetry test resulted in $\chi_{1}^{2}=22.59$ with $10 \mathrm{df}(p=.012)$ indicating only a moderate fit to the model. A closer look at the matchings with a relatively large contribution to $\chi_{1}^{2}$ led to the conclusion that in these cases the differences between observed and expected frequencies could be explained by a violation of the random guessing assumption.

The examinees appeared to respond to the questions based on a different domain of knowledge, in which the same concepts were used but with a different meaning. In the case of 1-e, the unconscious aspect is related to structuralism because the examinees thought of structuralism as a school within the domain of philosophy (Lacan, 1968) and cultural anthropology (Levi-Strauss, 1958). In this school, Freud's concept of the unconscious plays a central role. In the matching test, structuralism refers to Titchener's system, who opposed American functionalism. The unconscious aspect refers to the Würzburg School that interpreted thought as an unconscious process (see Leahey, 1980, p. 199). In the case of $4-d$, the examinee related the sensory aspect to phenomenology, because perceiving (the sensory aspect) implies perceiving phenomena. In the case of 5-e, the physiology aspect appears to be associated with functionalism because examinees related physiology to anatomy and then reasoned as follows: anatomy studies the structure of organs and physiology studies their function; therefore, physiology must be matched to functionalism. 
Table 9

Observed Frequencies and Frequencies Expected According to the KRG Model

\begin{tabular}{|c|c|c|c|c|c|c|c|c|c|c|c|c|}
\hline \multirow{2}{*}{$\begin{array}{l}\text { Question } \\
\text { Alternative }\end{array}$} & \multicolumn{6}{|c|}{$\begin{array}{l}\text { Observed Frequencies for } \\
\text { Response Alternatives }\end{array}$} & \multicolumn{6}{|c|}{$\begin{array}{c}\text { Expected Frequencies } \\
\text { for Response Alternatives }\end{array}$} \\
\hline & 1 & 2 & 3 & 4 & 5 & 6 & 1 & 2 & 3 & 4 & 5 & 6 \\
\hline \multicolumn{13}{|c|}{ Schools of Psychology } \\
\hline$d$ & 211 & 28 & 9 & $18^{*}$ & 1 & 3 & 211.0 & 24.0 & 13.0 & 13.0 & 3.0 & 6.0 \\
\hline$a$ & 20 & 200 & 11 & 10 & $11^{*}$ & 18 & 24.0 & 200.0 & 12.5 & 10.5 & 7.0 & 16.0 \\
\hline$e$ & $17^{*}$ & 14 & 206 & 21 & 5 & 7 & 13.0 & 12.5 & 206.0 & 22.5 & 6.5 & 9.5 \\
\hline$c$ & 8 & 11 & 24 & 200 & 16 & 11 & 13.0 & 10.5 & 22.5 & 200.0 & 13.5 & 10.5 \\
\hline$f$ & 5 & 3 & 8 & 11 & 211 & 32 & 3.0 & 7.0 & 6.5 & 13.5 & 211.0 & 29.0 \\
\hline$b$ & 9 & 14 & 12 & 10 & 26 & 199 & 6.0 & 16.0 & 9.5 & 10.5 & 29.0 & 199.0 \\
\hline \multicolumn{13}{|c|}{ Concepts in Social Psychology } \\
\hline$c$ & 132 & 7 & 13 & 1 & 19 & 18 & 132.0 & 5.0 & 13.5 & .5 & 25.0 & 14.0 \\
\hline$f$ & 3 & 156 & 6 & 3 & 12 & 10 & 5.0 & 156.0 & 8.5 & 2.5 & 9.5 & 8.5 \\
\hline$d$ & 14 & 11 & 127 & 0 & 22 & 16 & 13.5 & 8.5 & 127.0 & 0.0 & 23.0 & 18.0 \\
\hline$b$ & 0 & 2 & 0 & 183 & 4 & 1 & .5 & 2.5 & 0.0 & 183.0 & 3.5 & .5 \\
\hline$a$ & 31 & 7 & 24 & 3 & 51 & 74 & 25.0 & 9.5 & 23.0 & 3.5 & 51.0 & 78.0 \\
\hline$e$ & 10 & 7 & 20 & 0 & 82 & 71 & 14.0 & 8.5 & 18.0 & .5 & 78.0 & 71.0 \\
\hline \multicolumn{13}{|c|}{ Mathematics Needed for Psychology } \\
\hline$b$ & 54 & 18 & 5 & 10 & $24^{*}$ & 19 & 54 & 32.5 & 4 & 14 & 14 & 11.5 \\
\hline$e$ & $47^{*}$ & 33 & 5 & 10 & 24 & 11 & 32.5 & 33 & 7 & 11 & 33.5 & 13 \\
\hline$f$ & 3 & 9 & 48 & $45^{*}$ & 7 & 18 & 4 & 7 & 48 & 29.5 & 10.5 & 31 \\
\hline$d$ & 18 & 12 & 14 & 41 & 21 & 24 & 14 & 11 & 29.5 & 41 & 14.5 & 20 \\
\hline$c$ & 4 & 43 & 14 & 8 & 43 & 18 & 14 & 33.5 & 10.5 & 14.5 & 43 & 14.5 \\
\hline$a$ & 4 & 15 & $44^{*}$ & 16 & 11 & 40 & 11.5 & 13 & 31 & 20 & 14.5 & 40 \\
\hline
\end{tabular}

*Indicates a large contribution to $\chi_{1}^{2}$.

\section{Example 2: Concepts in Social Psychology}

This matching test was given to students $(N=190)$ as part of an examination in an introductory course on social psychology. The textbook (Brown, 1986) consisted of seven parts, but the examination was based only on the first three parts of the book. In order to be certain that at least some alternatives were not known by the students, two alternatives (question alternatives 5 and 6 ) were taken from part of the text not required for the examination. Students were told that the matching task only served research purposes and would not contribute to their final score. The response alternatives referred to theoretical concepts and the question alternatives to phenomena to be explained (see Table 8).

The observed and expected frequencies for the various matchings are given in Table 9. The resulting $\chi_{1}^{2}$ of 13.40 with $9 d f(p=.145)$ clearly indicates that the data behaved in accordance with the KRG model. The Guttman hypothesis was then tested, which implies that the examinees and the alternatives could be ordered on a Guttman scale. Table 10 shows a rearrangement of the observed frequencies according to the magnitude of the diagonal frequencies. The expected frequencies, also shown in Table 10, were computed according to the Guttman hypothesis.

The resulting $\chi_{2}^{2}$ of 43.87 with $20 \mathrm{df}(p=.002)$ was significant at the .01 level. However, this value of $\chi_{2}^{2}$ was completely determined by a single frequency ( 31 for alternative 1 in Table 10); the other observed frequencies did not severely deviate from their expected counterparts.

These mismatchings can be explained as follows. The examinees appeared to confound "evaluative consistency" with the "consistency" dimension in attribution theory. In attribution theory, "Consistency determines duration of the cause or causes of a behavioral event: They are either stable (high 
Table 10

Observed Frequencies and Frequencies Expected According to the KRG Model With an Underlying Guttman Scale for Theoretical and Empirical Concepts in Social Psychology

\begin{tabular}{|c|c|c|c|c|c|c|c|c|c|c|c|c|}
\hline \multirow{2}{*}{$\begin{array}{l}\text { Question } \\
\text { Alternative }\end{array}$} & \multicolumn{6}{|c|}{$\begin{array}{l}\text { Observed Frequencies for } \\
\text { Response Alternatives }\end{array}$} & \multicolumn{6}{|c|}{$\begin{array}{l}\text { Expected Frequencies } \\
\text { for Response Alternatives }\end{array}$} \\
\hline & 4 & 2 & 1 & 3 & 6 & 5 & 4 & 2 & 1 & 3 & 6 & 5 \\
\hline$b$ & 183 & 2 & 0 & 0 & 1 & 4 & 183.0 & 1.4 & 1.4 & 1.4 & 1.4 & 1.4 \\
\hline$f$ & 3 & 156 & 3 & 6 & 10 & 12 & 1.4 & 157.1 & 7.9 & 7.9 & 7.9 & 7.9 \\
\hline$c$ & 1 & 7 & 132 & 13 & 18 & 19 & 1.4 & 7.9 & 128.2 & 17.5 & 17.5 & 17.5 \\
\hline$d$ & 0 & 11 & 14 & 127 & 16 & 22 & 1.4 & 7.9 & 17.5 & 122.2 & 20.5 & 20.5 \\
\hline$e$ & 0 & 7 & 10 & 20 & 71 & 82 & 1.4 & 7.9 & 17.5 & 20.5 & 64.7 & 78.0 \\
\hline$a$ & 3 & 7 & $31^{*}$ & 24 & 74 & 51 & 1.4 & 7.9 & 17.5 & 20.5 & 78.0 & 64.7 \\
\hline
\end{tabular}

*Indicates a large contribution to $\chi_{2}^{2}$.

consistency) or unstable (low consistency)" (Brown, 1986, p. 146). Therefore, in this case the mismatching was not the result of some coping strategy, but must be considered as a genuine incorrect response. Evaluative consistency is a phenomenon that may be observed in impression formation. Therefore, the conclusion seems justified that an underlying Guttman scale definitely was appropriate for these data, but that the effect of it was distorted by a response bias with respect to response alternative 1 .

\section{Example 3: Mathematics Requirements for Psychology}

A matching task was given to freshmen $(N=130)$ in psychology to evaluate students' knowledge of elementary mathematics (see Table 8). The observed and expected frequencies are given in Table 9. The resulting $\chi_{1}^{2}$ of 85.76 with $10 d f(p<.001)$ is highly significant, indicating a poor fit to the KRG model. The following matchings had a relatively large contribution to $\chi_{1}^{2}: e-1, a-3, f-4$, and $b-5$. In these cases, there is a striking similarity between the matched alternatives; the examinees appeared to use some kind of coping strategy by looking for the most similar response alternative. This might explain the serious violation of the model.

\section{Discussion}

These results demonstrate that the KRG model does not always hold. The assumption of random guessing is easily violated, because examinees appear to use coping strategies when they do not know the correct answer. There are two alternatives to dealing with this problem. One possibility is to construct the alternatives in such a way that the various response alternatives are equally attractive. This can be achieved, for example, by using similar-appearing response alternatives or by avoiding socalled distractors. The latter, however, is probably less desirable for test constructors who usually prefer distractors for reasons of differential testing.

The other possibility is to use more complex models that take into account the use of coping strategies. For example, it might be the case that some type of knowledge, coping, or random guessing (KCRG) model applies. Let a given matching task consist of six question alternatives, $a, b, c, d$, $e, f$, and six response alternatives, $1,2,3,4,5,6$. Let the set of correct pairings be given by the set $\{(a, 1),(b, 2),(c, 3),(d, 4),(e, 5),(f, 6)\}$. Let the various coping strategies result in an examinee-independent set of one-to-one coping pairings: $\{(a, 2),(b, 5)\}$. The set of correct pairings is examinee-independent by definition. For each question alternative, three types of examinees are considered instead of the two types in the KRG model:

1. The examinee knows the correct pairing, denoted by $K$. 
2. The examinee does not know the correct pairing and decides to respond according to some coping strategy, denoted by $C$.

3. The examinee does not know the correct pairing and does not respond according to a coping strategy, but guesses at random, which is denoted by $G$.

For example, an examinee might answer the six questions with the following strategies:

Question: $a \begin{array}{llllll} & b & c & d & e & f\end{array}$

Strategy: C C K K G .

This examinee shows the following response behavior. For $d$ and $e$, the examinee will select response alternatives 4 and 5 , each with probability 1 . For $a$, the examinee will select response alternative 2 with probability 1. For $b$, the examinee would have selected response alternative 5 as a result of coping; however, because 5 has already been used as a response to $e$, the examinee will guess at random instead, selecting from the remaining response alternatives. As a result, the examinee will randomly match $b, c$, and $f$ to the response alternatives 1,3 , and 6 . The question alternatives, for which the correct response alternative is known are completed first. Then, the question alternatives for which the correct response alternatives are not known are completed using coping strategies. Response alternatives that were already used in the first stage cannot be used for the coping strategy. The question alternatives for which coping would apply, but is not possible, are further treated as alternatives for which random guessing applies.

According to this model, the following examinee patterns are also possible:

Question: $\quad a \quad b \quad c \quad d e f$

Examinee 2: $\mathrm{G} \mathrm{C} \mathrm{G} \mathrm{K} \mathrm{K} \mathrm{G}$

Examinee 3: C G G K K G

Examinee 4: G G G K K

Examinees 2 and 3 are inconsistent regarding their coping behavior. The model could be restricted by assuming that examinees similar to Examinees 2 and 3 would not occur. This might be the case when only one coping strategy is used, such as was the case in the mathematics task discussed above in which all coping pairings, $e-1, a-3, f-4$, and $b-5$, were based on similarity.

The intention of these examples was to illustrate realistic extensions of the KRG model, such as the KCRG model, that could be of interest in future applications. The KRG model can still be used despite the fact that it does not always account for the phenomena under study, because it can add insight into the actual choice mechanism used by the examinees. As illustrated above, this can be done by examining the $\chi_{1}^{2}$ contributions of the symmetry test. Large contributions refer to matchings that are preferred more than can be expected under the assumption of random guessing.

\section{References}

Abu-Sayf, F. K. (1977). A new formula score. Educational and Psychological Measurement, 37, 853-862.

Brown, R. (1986). Social psychology (2nd ed.). New York: The Free Press.

Budescu, D. V. (1988). On the feasibility of multiple matching tests. Applied Psychological Measurement, $12,5-14$.

Coombs, C. H., (1964). A theory of data. New York: Wiley.

Diamond, J., \& Evans, W. (1973). The correction for guessing. Journal of Educational Research, 43, $181-191$.

Hutchinson, T. P. (1982). Some theories of performance in multiple choice tests, and their implications for variants of the task. British Joumal of Mathematical and Statistical Psychology, 35, 71-89.

Kendall, M. A., \& Stuart, A. (1963). The advanced theory of statistics (Vol. 1). London: Griffin.

Lacan, J. (1968). The language of the self, the function of language in psychoanalysis. New York: Dell. 
Leahey, T. H. (1980). A history of psychology. Englewood Cliffs NJ: Prentice Hall.

Levi-Strauss, C. L. (1958). Anthroplogie structurale [Structural anthropology]. Paris: Plon.

Lord, F. M., \& Novick, M. R. (1968). Statistical theories of mental test scores. Reading MA: Addison-Wesley.

Van der Ven, A. H. G. S. (1980). Introduction to scaling. New York: Wiley.

Wood, D. A. (1960). Test construction. Columbus $\mathrm{OH}$ : Merrill Books.
Zimmerman, D. W., \& Williams, R. H. (1982). Element of chance and comparative reliability of matching tests and multiple-choice tests. Psychological Reports, 50, 975-980.

\section{Author's Address}

Send requests for reprints or further information to A. H. G. S. van der Ven, Department of Mathematical Psychology, University of Nijmegen, P.O. Box 9104, $6500 \mathrm{HE}$ Nijmegen, The Netherlands. 